\title{
Avaliação e atendimento psicológico na pandemia Covid-19 no Brasil: uma revisão sistemática
}

\author{
Assessment and psychological care in the Covid-19 pandemic in Brazil: a systematic
}

Evaluación y atención psicológica en la pandemia Covid-19 en Brasil: una revisión sistemática

Recebido: 09/09/2021 | Revisado: 15/09/2021 | Aceito: 21/09/2021 | Publicado: 23/09/2021

\author{
Milena de Oliveira Silva \\ ORCID: https://orcid.org/0000-0003-2408-4889 \\ Faculdade Irecê, Brasil \\ E-mail: milena.silvamyh@gmail.com \\ Adrielle Dionísio dos Santos \\ ORCID: https://orcid.org/0000-0003-4810-5218 \\ Faculdade Irecê, Brasil \\ E-mail: adrielledionisio@gmail.com \\ Victor Ronne Nunes De Souza \\ ORCID: https://orcid.org/0000-0001-6613-2135 \\ pela Faculdade Irecê, Brasil \\ E-mail: victor.ronne70@gmail.com \\ Erika Danielle Souza da Silva \\ ORCID: https://orcid.org/0000-0002-0954-9038 \\ Faculdade Irecê, Brasil \\ E-mail: erikadanielly20@gmail.com \\ Rodrigo Oliveira Damasceno \\ ORCID: https://orcid.org/0000-0002-0662-6827 \\ Universidade Federal de Pernambuco, Brasil \\ E-mail: digaodamasceno@yahoo.com.br
}

\begin{abstract}
Resumo
Objetivo: Compreender como estão sendo realizados os atendimentos e a avaliação psicológica em tempos de pandemia da Covid-19. Metodologia: Foi realizada uma revisão sistemática da literatura de abordagem quantitativa e qualitativa e natureza descritiva, cujo recorte temporal foi março de 2020 e abril de 2021, a busca de artigos nas bases de dados eletrônicas ocorreram entre os meses de março a abril de 2021. Resultados: No que se refere à quantidade de publicações relacionadas à temática, observa-se que, aparentemente, o crescente interesse científico nesta área está acontecendo a passos pequenos no Brasil, uma vez que foram selecionados 10 artigos científicos e 1 cartilha para compor a amostra final. Discussões: A prestação de serviços psicológico mediado por TICs representa um grande avanço para a ciência psicológica. No contexto de pandemia Covid-19, foi estabelecida a Resolução CFP n ${ }^{\circ}$ 04/2020 que regulamenta os serviços psicológicos prestados por TIC. No entanto, é preponderante avaliar de forma crítica essa nova forma de atendimento, uma vez que a mesma tende a permanecer após o período pandêmico e apresenta potencialidades, possibilidades e desafios. Considerações Finais: Em suma, o atendimento psicológico e a AP online vem sendo utilizados de maneira experimental a alguns anos, mas com o avanço da tecnologia, as demandas de tempo da sociedade e a pandemia da Covid-19 aumentou a procura por essa forma de atendimento. Entretanto, é necessário estudos mais robustos para demonstrar sua eficácia e eficiência, tal como de instrumentalização profissional para melhor atender as demandas, uma vez que, ainda há pouca publicação sobre a temática.
\end{abstract}

Palavras-chave: Covid-19; Avaliação psicológica online; Atendimento psicológico online.

\begin{abstract}
Goal: Understand how the attendance and psychological assessment are being taken during the Covid-19 pandemic. Methodology: It was made a systematic review of quantitative and qualitative approach literature and descriptive nature, whose temporal snippet was between March 2020 and April 2021, the search for articles in electronic databases happened between March and April 2021. Results: In what it refers to the amount of publications related to the topic, it is observed that, apparently, the growing scientific interest in this area is taking small steps in Brazil, since 10 scientific articles and 1 booklet were selected to compose the final sample. Discussions: The provision of psychological services mediated by TICs represents a great advance for psychological science. In the context of Covid-19 pandemic, it was established the Resolution CFP $\mathrm{n}^{\circ}$ 04/2020 that regulates the psychological services provided by TIC. Nonetheless, it is important to critically evaluate this new form of care, since the same tends to remais after the pandemic period and shows potential, possibilities and challenges. Final considerations: In short, psychological care and online AP have been used experimentally for some years, but with the advances of technology,
\end{abstract}


the society's time demand and the Covid-19 pandemic increased the search for this type of service. However, it's necessary robust studies for shows effectiveness and efficiency, such as professional instrumentation to better comply with the demands, since, there's still few publications about the subject.

Keywords: Covid-19; Online psychological assessment; Psychological support online.

\section{Resumen}

Objetivo: Comprender cómo se está llevando a cabo la atención y la evaluación psicológica en tiempos de la pandemia Covid-19. Metodología: Se realizó una revisión bibliográfica sistemática con un enfoque cuantitativo y cualitativo y de carácter descriptivo, cuyo marco temporal fue marzo de 2020 y abril de 2021, la búsqueda de artículos en bases de datos electrónicas se realizó entre marzo y abril de 2021. Resultados: Con respecto al número de publicaciones relacionadas con el tema, se observa que, aparentemente, el creciente interés científico en esta área se está dando a pequeños pasos en Brasil, ya que se seleccionaron 10 artículos científicos y 1 folleto para componer la muestra. Discusiones: La prestación de servicios psicológicos mediada por las TIC representa un gran avance para la ciencia psicológica. En el contexto de la pandemia Covid-19, se estableció la Resolución CFP No. 04/2020, que regula los servicios psicológicos que brindan las TIC. Sin embargo, es importante evaluar críticamente esta nueva forma de atención, ya que tiende a permanecer después del período pandémico y presenta potencial, posibilidades y desafíos. Consideraciones finales: En resumen, la atención psicológica y la AP en línea se han utilizado de manera experimental durante algunos años, pero con el avance de la tecnología, las demandas de tiempo de la sociedad y la pandemia Covid-19 ha aumentado la demanda de esta forma de asistencia. Sin embargo, se necesitan estudios más robustos que demuestren su efectividad y eficiencia, como la instrumentalización profesional para atender mejor las demandas, ya que aún existe poca publicación sobre el tema.

Palabras clave: Covid-19; Evaluación psicológica en línea; Atención psicológica en línea.

\section{Introdução}

\subsection{Psicologia, ciência e profissão: atendimento clínico e avaliação psicológica}

A psicologia caracteriza-se como uma ciência comprometida com a promoção da saúde mental através de uma atuação baseada no código de ética (CFP, 2005) e no respeito/busca da liberdade, dignidade, igualdade e integridade do ser humano. Logo, o profissional pode atuar nos setores da educação, saúde, lazer, do trabalho, da segurança, justiça, comunidades e comunicação (CFP, 2021).

Desta forma, segundo Fieldman (2015), a Psicologia é uma ciência que tem como foco o estudo do comportamento e dos processos mentais, onde o psicólogo pode atuar em distintas áreas, entre elas, está a prática clínica, a qual lida com o estudo, diagnóstico e tratamento de transtornos psicopatológicos. Correspondente a essa ideia, a psicologia clínica caracterizase como uma área específica da saúde, onde há uma busca por compreensão dos processos intra e interpessoais dos indivíduos (CFP, 2021).

Ainda segundo o CFP (2021), a atuação clínica tem como foco principal a prevenção e/ou cura da demanda trazida pelo cliente, para tal, a atuação dos profissionais pode ocorrer de modo isolado ou com o apoio de uma equipe multiprofissional em instituições. Ademais, o atendimento clínico realiza diagnóstico e acompanhamento psicológico, pesquisa e intervenção psicoterápica e utilizando distintas abordagens teóricas.

Para alcançar os objetivos propostos pelo atendimento, o profissional da psicologia pode fazer o uso da Avaliação Psicológica (AP). Essa é definida na resolução n ${ }^{\circ}$, emitida pelo CFP de 25 em abril de 2018, no Art. $1^{\circ}$, como a investigação de fenômenos psicológicos, de modo estruturado, utilizando distintos métodos, técnicas e instrumentos. A AP tem como objetivo a coleta de informações para elaboração de uma intervenção a partir da especificidade existente na demanda (CFP, 2021; Gouveya, 2018; Pasquali, 2016).

Complementando essa ideia, Cohen, Swerdlik e Sturman (2014) chamam atenção para a diferença existente entre testagem e AP. A testagem se caracteriza pelo uso de instrumentos e procedimentos projetados para mensurar variáveis do comportamento humano, focando apenas nos resultados, enquanto a AP tem como base a coleta e a associação de dados, por meio do uso de testes, entrevista, estudos de caso e observação, especificamente projetados para atender a demanda. Isto posto, 
a testagem se caracteriza como uma parte da AP.

Diante dessa ideia, a Cartilha de Avaliação de Psicológica, destaca que a realização de uma AP pode ter como foco o diagnóstico, planejamento de intervenções e acompanhamento, podendo ser aplicada no contexto da psicologia clínica; da saúde e hospitalar; na escolar e educacional; no âmbito jurídico; no esporte, no trânsito, seu uso, ainda pode ser estendido a área social e comunitária, na neuropsicologia, nas organizações e para orientação profissional, entre outros contextos (CFP, 2021).

Logo, para a realização de uma AP se faz necessário a análise de critérios preestabelecidos como: o cadastro do teste no Sistema de Avaliação de Testes Psicológicos (SATEPSI) e com o parecer favorável pelo CFP, a análise da demanda e a finalidade do teste. Além disso, o profissional precisa ter qualificação profissional na área, contar com um ambiente adequado para a aplicação da AP, fazer a guarda de documentos de modo correto. Deve também, disponibilizar as informações da avaliação para os indivíduos e garantir a proteção e integridade dos testes, defendendo o uso exclusivo da psicologia e a não comercialização de testes psicológicos (CFP, 2021; Borsa, 2016).

Consoante a essa ideia, Hutz, Bandeira e Trentini (2015) afirmam que, na aplicação e interpretação de resultados em uma AP, deve ser levado em consideração princípios básicos tal como, o respeito pela pessoa - considera a autonomia dos indivíduos e seus direitos; a beneficência, onde a AP deve ser realizada para beneficiar o avaliado, quando este critério não puder ser atendido o psicólogo pode tomar as decisões necessárias para manter os padrões éticos em seu trabalho. Outro princípio importante é a justiça, onde os testes utilizados devem estar adaptados à realidade biopsicossocial dos sujeitos, caso esse critério não seja levado em consideração os resultados da avaliação podem ser invalidados.

No caso de solicitação de resultados da AP, o psicólogo deve construir um documento, este deve estar de acordo com os princípios da Resolução nº 6, de 29 de março de 2019 emitida pelo CFP. Tal documento tem o objetivo de regulamentar, instituir regras e orientar a escrita do profissional, tendo como base o Código de Ética (CFP, 2005) e a análise histórica e social do indivíduo, fatores esses, que podem influenciar o processo de avaliação. A resolução emitida, também traz informações sobre os tipos de documentos que são produzidos, a finalidade e modo como deve ocorrer a devolutiva.

Frente ao que foi exposto, nota-se que a psicologia é uma ciência regulamentada e a atuação do psicólogo se estende a diferentes contextos, com diferentes abordagens e pode ter um foco individual ou grupal, tendo o apoio de outros profissionais em contextos específicos. Desse modo, a atividade do profissional antes presencial se estendeu para o atendimento online, abrangendo a atuação do profissional e promovendo a construção de uma reflexão teórica, metodológica e prática capaz de suprir a nova demanda emergente.

\subsection{Novas formas de atendimento: psicologia e atendimento psicoterápico online}

Diante da sociedade contemporânea e o avanço das tecnologias, novas formas de comunicação e de relações surgem, dando espaço para os atendimentos online em diversos âmbitos, incluindo o tratamento psicoterápico. Sendo assim, o atendimento psicológico online pode ser feito a partir de mensagens, seja de texto ou audiovisual simultâneo, utilizando programas de comunicação e e-mails (Ulkovski, Silva, \& Ribeiro, 2017).

Desse modo, em visão do mundo atual, o atendimento psicológico online pode ser considerado um avanço que acompanha as demandas da vida moderna, e nesses casos, pode ser uma excelente solução, já que permite que se tenha acesso a profissionais capacitados com os mesmos benefícios da terapia presencial. Tornando-se uma opção prática, segura e eficiente que permite ao paciente iniciar o tratamento e cuidar da sua saúde mental e emocional sem precisar mudar toda a sua rotina (Ulkovski, 2017; CFP, 2020).

Durante décadas de estudos, a psicologia vem demonstrando através da prática e da pesquisa, a utilização de diferentes ferramentas como forma de estabelecer o vínculo terapêutico com o cliente, tendo como estabelecimento da relação 
terapêutica ou rapport, algo fundamental para o progresso do paciente em seu tratamento. Além disso, em diferentes teorias psicológicas como a Terapia Cognitivo Comportamental (TCC), o terapeuta se mantém interativo com o cliente, logo, no atendimento online as possibilidades de atendimento são diversas, assim como de maneira presencial, sendo essa prática regulamentada pelo CFP através da Resolução n 011/ 2018 (Siegmund \& Lisboa, 2015).

O CFP (2018) através da resolução ${ }^{\circ}$ 011/ 2018, revogou a Resolução CFP $n^{\circ} 11 / 2012$ que regulamentava o atendimento psicológico de caráter experimental utilizando tecnologias que permitem comunicação a distância. Agora, é possibilitado a realização de AP online, utilizando da testagem favorável ao SATEPSI sendo específico para essa forma de atendimento.

A partir daí, com resolução n $011 / 2018$, fica regulamentada a prestação de serviço online feito por profissionais da psicologia, de maneira ética. Assim, o atendimento pode ser síncrono em que as duas ou mais pessoas se encontram em tempo real, ou assíncrono, que não é necessário a conexão simultânea, o psicólogo pode ler mensagens, e-mails dos pacientes ao longo do dia, sendo oferecido ao profissional diversas formas de atendimento remoto (CFP, 2018; Ulkovski, 2017; Gomes, 2011).

Em detrimento disso, utilizando as ferramentas regulamentadas, a resolução $\mathrm{n}^{\circ}$ 011/ 2018 autorizou os seguintes serviços psicológicos para serem realizados utilizando meios Tecnológicos de Informação e Comunicação (TICs): Atendimentos psicológicos e consultas de diferentes tipos, seleção de pessoal, utilização de instrumentos psicológicos regulamentados e testes favorável do SATEPSI e supervisão técnica dos serviços prestados por psicólogos em diferentes atuações (CFP, 2018).

Assim sendo, com o novo contexto de atendimento, no ano de 2020 foi estabelecida a Resolução CFP no 004/2020, regulamentando o trabalho prestado pelo psicólogo no contexto da pandemia da COVID-19 por meio das TICs. Dessa forma, é requisitado do psicólogo o cadastro na plataforma E-psi vinculado a seu Conselho Regional de Psicologia (CRP), devendo ter seu cadastro sempre atualizado, e não prestar atendimento por meio das TICs sem a devida autorização (CFP, 2008).

\subsection{Pandemia da Covid-19 e a emergência do atendimento e avaliação psicológica online}

Devido aos avanços tecnológicos e o período de pós-modernidade (Bauman, 2007), no qual a internet foi um dos marcos, o atendimento online surgiu como aliado aos profissionais para auxiliar na sua atuação. Diante disso, a psicologia enquanto campo de conhecimento, ciência e profissão, buscou se adequar às demandas trazidas pela modernidade, a exemplo do uso das TICs como ferramenta de trabalho. Essa demanda foi suscitada com o surgimento da pandemia da Covid-19 no final de 2019.

De acordo com Ornell et al. (2020), em 31 de dezembro de 2019, em Wuhan, na China, foi identificada uma nova doença respiratória caracterizada por um estado clínico que oscila de infecções assintomáticas a situações respiratórias graves. Essa doença é ocasionada pelo vírus Coronavírus SARS-CoV-2 (Covid-19) e possui um elevado poder de contágio, contribuindo para instaurar um período marcado por medo, incertezas quanto ao futuro, mudança social e comportamental (Oliveira et al., 2020).

Conforme notificação da Organização Mundial da Saúde (OMS) e Ministério da Saúde (MS) (2020), a sua via de transmissão ocorre por meio de gotículas respiratórias, tanto pelo contato direto, quanto pelo contato indireto com pessoas infectadas. Igualmente, o vírus pode ser contraído ao tocar o rosto com as mãos após contato com objetos ou superfícies contaminadas. Vale ressaltar que pessoas infectadas podem permanecer assintomáticas e transmitindo o vírus a outras. Assim, devido à rápida escalada da doença e disseminação a nível global, a OMS declarou pandemia em março de 2020 (Wang et al., 2020; Xiao, 2020).

Diante disso e com a falta de conhecimento a respeito deste agente viral e o alto índice de contágio, as autoridades 
públicas e sanitárias adotaram o isolamento social como meio de prevenção, tentando assim, diminuir o número de acometidos pela doença (Silva \& Ribeiro, 2020). Com essa forma de prevenção, os eventos festivos, aglomerações, academias, igrejas e atividades laborais consideradas não essenciais foram proibidas em diversas partes do mundo.

Nesse ínterim, os setores empregatícios tiveram que parar suas atividades por um período e procurar formas de se adaptar à nova situação decretada, uma vez que existia a incerteza de quanto tempo duraria a pandemia e quando poderia exercer suas atividades presenciais novamente. Com essas restrições impostas, surge a necessidade de readaptar-se e se adequar às novas formas de atendimento, sendo a opção remota e a online as mais utilizadas, através das TICs.

Nesse cenário pandêmico, com as incertezas geradas pela pandemia e a necessidade emergente de atendimento psicológico à população, conforme dados do CRP da $3^{\text {a }}$ Região (2020), a procura por atendimento psicológico online na plataforma E-psi, aumentou $800 \%$, sofrendo aumentos significativos na medida em que os períodos de isolamento social ficaram mais longos (Viana, 2020). Diante disso, o CFP (2020) enquanto órgão regulamentador da profissão, utilizando de seus poderes, publica em 26 de março de 2020, em caráter emergencial, a resolução nº 04.

Essa resolução dispõe sobre a regulamentação dos serviços psicológicos prestados por meios das TICs durante a pandemia da Covid-19, trazendo em seus artigos informações pertinentes a esse formato de atendimento de forma esporádica e autorizando a prestação desses serviços, incluindo a AP. Para tanto, o profissional deve realizar um cadastro prévio na plataforma E-Psi junto ao CRP e utilizar as recomendações do Código de Ética Profissional (CFP, 2005) e as resoluções pertinentes à modalidade de atendimento (CFP, 2020a).

Dessa forma, com a pandemia da Covid-19 e a necessidade de isolamento social, muitos profissionais da psicologia que atuam presencialmente, tiveram que se adaptar a modalidade online para que seus atendimentos fossem mantidos. Sendo assim, o psicólogo em sua autonomia e responsabilidade profissional precisou analisar caso a caso e viabilizar a continuidade dos serviços, identificando qual meio de comunicação seria ideal para cada um. Logo, para que os atendimentos aconteçam de forma fidedigna, algumas orientações precisam ser seguidas pelo profissional para que seus serviços migrem para a modalidade online. Alguns deles, segundo CFP (2018), é que após o cadastro e aprovação no E-Psi, a plataforma do CFP define qual ferramenta será utilizada para o atendimento levando em conta todo o conhecimento do profissional e do cliente, também se faz necessário a garantia do sigilo e qualidade do serviço de ambas as partes.

O CFP (2018) pontua ainda que é de extrema importância formalizar a prestação do serviço através de um contrato onde devem estar estabelecidos os direitos e deveres de ambas as partes, incluindo questões como a natureza do serviço, forma de atendimento, duração, frequência, sigilo, cuidados, remuneração e demais questões pertinentes e peculiares a cada situação. No site do CRP existem alguns modelos disponíveis e os mesmos podem ser adaptados de acordo com a necessidade da demanda do profissional.

Já no que se refere a AP realizada online, o CFP (2018) propõe algumas recomendações. Do mesmo modo dos processos de AP realizadas presencialmente, a prática por meio das TICs deve seguir as especificações técnicas e éticas impostas pelo CFP, com uma adição de cuidados específicos para que ocorra de forma segura, sigilosa, eficaz e ética (CFP, 2018; McCord et al., 2020; Marasca et al., 2020).

Segundo Marasca et al. (2020), é importante que o profissional antes de iniciar a AP online conheça a demanda e a pertinência para um processo em modalidade remota, ponderando, desse modo, os benefícios e possíveis prejuízos. De acordo com Luxton, Pruitt e Osenbach (2014), devem ser levadas em conta as características do sujeito atendido, fatores culturais, familiaridade com o uso da tecnologia e a disponibilidade de acesso a aparelhos eletrônicos, tal como a qualidade da conexão da internet, a fim de que sejam empregadas técnicas mais adequadas.

Além disso, cabe ao profissional analisar as técnicas a serem utilizadas, principalmente no que se refere aos testes disponíveis no mercado para a testagem de forma virtual, junto ao SATEPSI. Outro aspecto que deve ser levado em 
consideração é o baixo controle do psicólogo acerca do ambiente de avaliação e possíveis interrupções. O profissional deve se atentar e inteirar o avaliando sobre quais informações serão repassadas e quem terá acesso a elas, reforçar o sigilo, alertando para a não autorização de gravações de áudio e vídeo, bem como formalizar um contrato por escrito, em que essas recomendações, além de explicadas, estejam aceitas pelo avaliado (Lustgarten \& Elhai, 2018; CFP, 2018, AERA, APA \& NCME, 2014).

Em suma, diante dos dados apontados, observa-se que a regulamentação da prestação de serviços psicológicos online contribuiu para ampliar as possibilidades de atuação do psicólogo. A prestação de serviços psicológicos por meio das TICs, tem suas vantagens e desvantagens, cabendo ao profissional da psicologia, a psicologia enquanto campo de conhecimento, ciência e profissão e aos conselhos Regionais e Federal munir os profissionais com instrumentos e técnicas mais adequadas que os auxiliem, levando em consideração a ética e a cientificidade.

Nesse sentido, a finalidade deste estudo é compreender como estão sendo realizados os atendimentos e a avaliação psicológica em tempos de pandemia da Covid-19, o qual é justificado pela apresentação de informações científicas relevantes para ciência psicológica e a atuação do profissional da psicologia, tal como para a clientela. Para tal, utilizou-se de revisão sistemática da literatura de abordagem quantitativa e qualitativa e natureza descritiva, cujo recorte temporal foi março de 2020 e abril de 2021 e a busca nas bases de dados eletrônicas ocorreram entre os meses de março a abril de 2021.

\section{Metodologia}

O presente estudo trata-se de uma revisão sistemática da literatura, definida como um apanhado dos resultados de pesquisas, selecionados de maneira sistematizada e relacionado com a problemática específica, sendo útil na integração de informações sejam elas conflitantes ou coincidentes (Sampaio \& Mancini, 2007; Souza \& Ribeiro, 2009; Brasil, 2012;).

A mesma foi realizada a partir de levantamento bibliográfico eletrônico nas seguintes bases de dados: Scientific Electronic Library Online (Scielo); Portal de Periódicos Eletrônicos em Psicologia (PePSIC); Google Acadêmico; Medical Literature Analysis and Retrievel System Online (Medline); Research, Society and Development; Biblioteca Virtual em Saúde (BVS); Biblioteca Virtual em Saúde - Psicologia (BVS-Psi); Sistema de Bibliotecas e Informações da UFRJ.

Diante disso, a pesquisa apresenta abordagem quantitativa e qualitativa e teor descritivo, sendo construída através da adaptação das 8 etapas definidas por Brasil (2012): elaboração da pergunta norteadora de investigação; produção de protocolo de investigação; elaboração de critérios de elegibilidade; estratégias de pesquisa e busca na literatura; coleta de dados; análise crítica dos estudos incluídos; extração de dados; discussão dos resultados e apresentação da revisão.

Executou-se o estudo a partir da seguinte pergunta norteadora: Como estão sendo realizados os atendimentos online e a avaliação psicológica em contexto de pandemia da Covid-19? Para orientar a formulação da questão de pesquisa de forma clara e objetiva, optou-se por estruturá-la segundo os componentes do acrônimo PICO (Brasil, 2012), no qual P representa População, I - Intervenção, C - Controle e O - Desfecho. Nesse caso, usou-se PIO, uma vez que não houve o estabelecimento de comparador ou controle definido, a qual P refere-se a psicólogos e clientes, I - atendimento e avaliação psicológica online e O: adesão dos atendimentos psicológicos por meio da TIC.

A técnica de busca foi efetuada através dos cruzamentos dos Descritores em Ciências de Saúde (DeCs); Avaliação psicológica online; Serviços psicológicos/Intervenções psicológico/Atendimento psicológico online/psicoterapia online e tecnologias da informação e comunicação, Pandemia da Covid-19, com cruzamento aleatório entre os descritores conectados pelo operador booleano AND.

Além disso, realizou-se a seleção das evidências científicas a partir da adesão das referências dentro do recorte temporal de janeiro de 2020 a abril de 2021 e tendo critérios de elegibilidade, sendo considerados critérios de inclusão: 1) Os 
artigos devem discutir a avaliação e/ou atendimento psicológico online na pandemia da Covid-19;2) devem ser trabalhos publicados e disponíveis integralmente em bases de dados científicas; 3) Serão incluídos normas técnicas, cartilhas e portarias publicadas pelos Conselhos Regionais e Federal de psicologia que autorizam o atendimento online na pandemia da Covid-19; e os critérios de exclusão: 1) Serão excluídos trabalhos que não estão em língua portuguesa; 2) Serão excluídos livros, teses e artigos não científicos (informações de blog, notícias). A pesquisa em bancos de dados eletrônicos foi realizada entre os meses de março e abril de 2021.

No início da pesquisa identificou-se 67 arquivos em sua totalidade na busca em base de dados, sendo 65 artigos científicos e 2 cartilhas de recomendações e orientações aos profissionais. Ao realizar a primeira triagem foram excluídos 21 trabalhos duplicados. Das 46 publicações restantes foram excluídas 3 devido às mesmas não estarem em língua portuguesa e 20 por não se adequarem ao recorte temporal definido para a amostra - janeiro de 2020 a abril de 2021.

Após, realizou-se a leitura dos títulos e resumos dos artigos para realizar uma triagem destas referências e descartar um grande número de estudos que não se enquadram nos critérios de elegibilidade estabelecidos pela revisão (Brasil, 2012). Desse modo, restaram 23 estudos para a aplicação dos critérios de inclusão e exclusão, dos quais, após leitura do título e resumo simples foram incluídos para o banco de dados final 10 artigos científicos $(90,9 \%)$ e 1 cartilha $(9,1 \%)$, para análise e realização de revisão, conforme evidencia-se no Figura 1.

Figura 1: Detalhadamente da busca dos artigos científicos em base de dados.
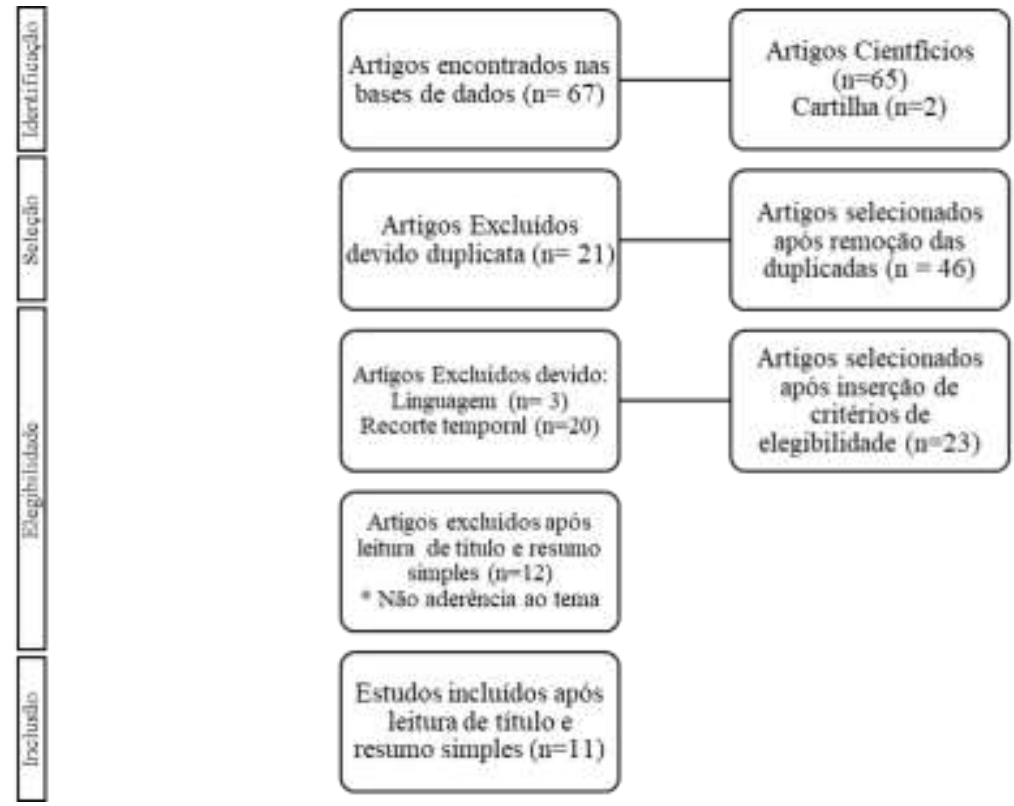

Fonte: Figura adaptado de PRISMA.

Ressalta-se que houve o preenchimento de checklist previamente padronizado em Microsoft Word por dupla de autores para diminuir o viés da pesquisa, com base nos critérios de elegibilidade, para tabulação dos resultados obtidos.

\section{Resultados}

No que se refere à quantidade de publicações relacionadas à temática em questão, observa-se que, aparentemente, o crescente interesse científico nesta área está acontecendo a passos pequenos no Brasil. Dos estudos selecionados para comporem a amostra final, 10 artigos foram publicados no ano de 2020 (90,9\%) e apenas em 1 de 2021 (9,1\%). Desses, 10 
$(90,9 \%)$ são artigos científicos e 1 cartilha $(9,1 \%)$. As publicações que compuseram a amostra final foram organizadas no

Quadro 1, a qual apresenta as características que foram avaliadas neste estudo.

Quadro 1: Estudos incluídos na amostra final.

\begin{tabular}{|c|c|c|}
\hline TÍTULOS & AUTORIA & OBJETIVO \\
\hline $\begin{array}{l}\text { Psicoterapia online: uma revisão de } \\
\text { literatura }\end{array}$ & Bittencour et al. (2020) & $\begin{array}{c}\text { Compreender os conceitos envoltos no processo terapêutico } \\
\text { online. }\end{array}$ \\
\hline $\begin{array}{l}\text { Aconselhamento psicológico } \\
\text { fenomenológico-existencial online } \\
\text { como possibilidade de atenção } \\
\text { psicológica durante a pandemia de } \\
\text { Covid-19 }\end{array}$ & $\begin{array}{l}\text { Evangelista \& Cardoso } \\
\qquad(2020)\end{array}$ & $\begin{array}{l}\text { Busca-se um entendimento deste momento, sob a perspectiva } \\
\text { fenomenológico-existencial, como de ruptura com a } \\
\text { familiaridade cotidiana, que lança a existência na } \\
\text { indeterminação e na busca de modos de reestabilização. }\end{array}$ \\
\hline $\begin{array}{l}\text { Os desafios dos serviços psicológicos } \\
\text { mediados pelas TIC no contexto da } \\
\text { pandemia do coronavírus 2019-2020 }\end{array}$ & Santana et al. (2020) & $\begin{array}{c}\text { Reflexão crítica sobre a tecnologia visualizando os } \\
\text { atendimentos psicológicos mediados pelas Tecnologias da } \\
\text { Informação e Comunicação no contexto da Pandemia do } \\
\text { Coronavírus 2019-20 mentalizando a reconfiguração e/ou } \\
\text { rompimento de paradigmas/obstáculos a partir de seus usos na } \\
\text { Psicologia. }\end{array}$ \\
\hline $\begin{array}{l}\text { Terapia Online com Casais e Famílias: } \\
\text { Prática e Formação na Pandemia de } \\
\text { Covid-19 }\end{array}$ & Schmidt et al. (2020 & $\begin{array}{c}\text { Sistematizar conhecimentos sobre terapia online com casais e } \\
\text { famílias, trazendo considerações para a prática e a formação } \\
\text { profissional diante da pandemia }\end{array}$ \\
\hline $\begin{array}{l}\text { Entrevista Online: Potencialidades e } \\
\text { desafios para coletas de dados no } \\
\text { contexto da pandemia de Covid }-19\end{array}$ & Schmidt et al. (2020) & $\begin{array}{l}\text { Apresentar as potencialidades e os desafios da realização de } \\
\text { entrevistas online no contexto da pandemia de Covid-19 }\end{array}$ \\
\hline $\begin{array}{l}\text { Atendimento Psicológico Online: } \\
\text { Desafios que a covid-19 convida a } \\
\text { Transpor na Clínica psicanalítica }\end{array}$ & $\begin{array}{l}\text { Salek, Palazzi \& } \\
\text { Piccinini (2020) }\end{array}$ & $\begin{array}{l}\text { Observar as possibilidades destes fazeres e suas possíveis } \\
\text { contribuições neste momento singular que se faz presente }\end{array}$ \\
\hline $\begin{array}{l}\text { Cartilha de boas práticas para avaliação } \\
\text { psicológica em contextos de pandemia }\end{array}$ & CFP (2020) & $\begin{array}{l}\text { Apresentar informações para a promoção de uma atuação } \\
\text { profissional respaldada nos preceitos éticos contidos no Código } \\
\text { de Ética Profissional do Psicólogo (2005), respeitando a } \\
\text { confidencialidade necessária às práticas específicas da AP. }\end{array}$ \\
\hline $\begin{array}{l}\text { Saúde mental e intervenções } \\
\text { psicológicas diante da pandemia do } \\
\text { novo coronavírus (Covid-19) }\end{array}$ & Schmidt et al. (2020) & $\begin{array}{c}\text { Sistematizar conhecimentos sobre implicações na saúde mental } \\
\text { e intervenções psicológicas diante da pandemia do novo } \\
\text { coronavírus }\end{array}$ \\
\hline $\begin{array}{l}\text { Plantão psicológico: a prática do } \\
\text { acolhimento online durante a pandemia } \\
\text { da Covid- } 19\end{array}$ & Pereira et al. (2021) & $\begin{array}{c}\text { Relatar a experiência de um projeto de extensão realizado pelo } \\
\text { Serviço Escola de Psicologia de uma universidade pública de } \\
\text { Minas Gerais, que objetivou ofertar atendimento psicológico à } \\
\text { comunidade acadêmica durante a pandemia da Covid-19, no } \\
\text { período de abril a julho de } 2020\end{array}$ \\
\hline $\begin{array}{l}\text { Atendimento Psicológico online no } \\
\text { Contexto da Pandemia de Covid-19 }\end{array}$ & Viana (2020) & $\begin{array}{l}\text { Discutir os diversos e complexos fatores que envolvem a } \\
\text { prestação de serviços psicológicos e de saúde mediados por } \\
\text { Tecnologias da Informação e da Comunicação (TICs), também } \\
\text { conhecidos como atendimentos online }\end{array}$ \\
\hline $\begin{array}{l}\text { Avaliação psicológica online: } \\
\text { considerações a partir da pandemia do } \\
\text { novo coronavírus (Covid-19) para a } \\
\text { prática e o ensino no contexto a } \\
\text { distância }\end{array}$ & Marasca et al. (2020) & $\begin{array}{l}\text { Discutir a viabilidade de processos de Avaliação Psicológica } \\
\text { online e apontar direções para seu aperfeiçoamento }\end{array}$ \\
\hline
\end{tabular}

Fonte: Autores.

Como pode ser observado na tabela acima, dos $10(90,1 \%)$ artigos selecionados, apenas $1(10 \%)$ discute sobre a AP online, $8(80 \%)$ buscam o entendimento sobre o atendimento psicológico online e $1(10 \%)$ discute acerca de intervenções em saúde mental durante a pandemia da Covid-19. Dos 8 artigos, 1 (12,5\%) refere-se a um relato de experiência sobre o atendimento psicológico online, $1(12,5 \%)$ os conceitos envolvidos na psicoterapia online, $3(37,5 \%)$ retratam sobre os 
desafios dessa modalidade de atendimento, $2(25 \%)$ as potencialidades e $1(12,5 \%)$ apresenta sobre atendimento psicológico online com casais.

Constata-se também que dentre a quantidade total das evidências científicas identificadas, um estudo intitulado "Avaliação psicológica online: considerações a partir da pandemia do novo coronavírus (Covid-19) para a prática e o ensino no contexto a distância" foi encontrado 7 vezes, o que reverbera a necessidade de mais estudos sobre a temática e a importância de discussões mais abrangente.

A cartilha selecionada $(9,1 \%)$, por sua vez, apresenta discussões ricas sobre a prestação de serviços psicológicos online trazendo recomendações importantes ao profissional da psicologia acerca da prática específica da AP em contexto de pandemia, visando dar continuidade às atividades do ensino, prática e uso de testes psicológicos em tempos de pandemia. Ressalta-se a necessidade do profissional está atento às orientações e buscar atualizações constantes no que se refere à atuação profissional nos diferentes formatos.

\section{Discussões}

\subsection{Normatizações e padronização do atendimento e da avaliação psicológica online}

A psicologia como prática profissional, se inseriu nesse contexto de atendimento remoto tendo como base a Resolução CFP no 011/2018, ampliando as possibilidades sobre os serviços prestados pelos psicólogos utilizando TICs. Além disso, a resolução trouxe a necessidade do cadastro dentro dos Conselhos Regionais (CRP) e do Conselho Federal (CFP) na plataforma E-psi para um maior controle da fundamentação da tecnologia, serviços, públicos e os limites do uso dessa ferramenta (Viana, 2020).

Na Resolução do CFP nº 011/2018 a AP foi regulamentada como uma possibilidade de realização remota, utilizando testes específicos padronizados e normatizados para esse tipo de atendimento. Tal como a AP realizada presencialmente, a prática utilizando as TIC deve seguir pormenorizações técnicas e éticas específicas (Marasca et al., 2020).

Com isso e com as mudanças recentes da Resolução do CFP n $n^{\circ}$ 011/2018, segundo Viana (2020), a procura por psicólogos para a realização do cadastro na plataforma E-psi cresceu exponencialmente, também em detrimento do contexto atual da pandemia da Covid-19. Nesse ínterim, foi estabelecida a Resolução CFP no 04/2020, regulamentando os serviços psicológicos prestados por TIC durante a pandemia da Covid-19.

Dessa forma, com o aumento do número de profissionais atuando remotamente, e com a crescente demanda pela AP e terapia online, são necessárias mais ferramentas e especializações para o psicólogo que deseja se aperfeiçoar nessa prática. Acerca disso, além do conhecimento sobre a teoria psicológica aplicada é preciso entender o básico sobre a tecnologia e as linguagens, para construção de um conhecimento de fácil acesso (Santana et al., 2020).

\subsection{Avaliação Psicológica Online: das potencialidades as possibilidades}

A AP online, tendo passado por debates e transformações no decorrer dos anos, possibilitou aos profissionais da psicologia a construção de um novo saber prático, onde se busca uma atuação ética e benéfica para o cliente. Assim, nota-se os benefícios do atendimento remoto bem como as possibilidades para a psicologia ao utilizar as TICs.

Diante disso, Bittencurt et al. (2020) apontam que o uso das TICs possibilita ao profissional uma atuação além do espaço físico, diminuindo a distância e otimizando o tempo, além disso, serve como uma nova forma de estabelecer uma conexão com as pessoas. Ainda segundo os autores, pesquisas realizadas sobre os resultados e a eficácia da psicoterapia online para o cliente é similar a psicoterapia presencial.

O modelo online também apresenta vantagens como o acesso mais amplo, - podendo atingir diferentes públicos - a 
diminuição dos custos e pode ter maior receptividade de clientes que apresentam limitações. Nota-se ainda, que o uso de técnicas online para determinados transtornos e contexto podem ser mais benéficos, uma vez que, possibilita o alcance de melhor resultados nas demandas que chegam até o psicólogo (Bittencourt et al., 2020; Schimidt, et.al., 2020).

O Atendimento online, pode ser utilizado em diversos contextos e áreas, entre eles pode ser citado a terapia online com casais e famílias, que segundo Schimidt et al. (2020), há múltiplos benefícios, como a facilidade que alguns clientes apresentam para estabelecer um vínculo com o terapeuta, a capacidade de juntar membros que se encontram distantes e regiões com poucas possibilidades de atendimento. Para tal, se faz necessário a atuação de um profissional capacitado que tenha desenvolvido habilidades para lidar com o meio digital e seus respectivos empecilhos.

Além das vantagens listadas, os atendimentos mediados pelas TICs podem beneficiar os serviços públicos e privados, auxiliando na construção de um conhecimento prático, teórico e metodológico, podendo aumentar a troca de informações entre profissionais e polos de pesquisa no âmbito psicológico. Diante disso, Viana (2020) ressalta a necessidade de estar atento aos cuidados que devem ser tomados em questões éticas.

Complementando essa ideia, de acordo com Marasca et al. (2020), no caso da prática e ensino da AP na pandemia da COVID-19, permite uma aproximação de profissionais e supervisores, garantindo a manutenção das supervisões caso não possa ser realizada de modo presencial. Em sequência, na supervisão clínica, diversas plataformas podem auxiliar e dar suporte para que o trabalho do psicólogo atenda a demanda trazida pelo cliente (Gouveia \& Mouser, 2020).

Isto posto, é possível compreender que a AP online, no contexto da pandemia da Covid-19, apresenta diversas vantagens e benefícios tanto para os profissionais quanto para os clientes, podendo ser utilizada em diversas áreas da psicologia. Assim, o profissional conta com um leque de atividades que podem ser trabalhadas via TICs.

\subsection{Os possíveis desafios encontrados diante do atendimento e da avaliação psicológica online}

A prestação de serviços psicológicos mediado por TICs representa um grande avanço para a ciência psicológica. No entanto, é preponderante avaliar de forma crítica essa nova forma de atendimento, uma vez que tende a permanecer após o período pandêmico, apresentando tanto potencialidades, quanto desafios, e a estes, a ciência psicológica deve buscar meios de suprir para melhor adequação e eficácia dos serviços prestados.

De acordo com Bittencour et al. (2020), a terapia online cria mecanismos mais fáceis e acesso às pessoas que por algum motivo não conseguem sair de casa, tendo no atendimento mediado pela tela, sustentação, continências e possibilidades de transformação da experiência emocional. Entretanto, com a prestação de serviços online, o profissional de psicologia passou a enfrentar desafios, como o conhecimento sobre as TIC e a necessidade de se munir com métodos, técnicas, procedimentos e instrumentos adequados a esse novo contexto (CFP, 2020b).

Dentre os desafios, encontra-se a qualidade na conexão de internet do cliente e do profissional, assim como as interrupções ocorridas durante o atendimento psicológico que podem impactar diretamente no processo terapêutico. Além disso, o ambiente em que o paciente se encontra para participar da sessão de terapia também tende a se apresentar como fator de risco para o não estabelecimento de uma boa relação terapêutica, tal como a familiaridade do cliente e do profissional com a ferramenta tecnológica a ser utilizada para o atendimento, a disponibilidade de acesso a aparelhos eletrônicos por parte do cliente, habilidades e competências no manejo dessas tecnologias e a segurança do ambiente virtual a ser utilizado (CFP, 2020b).

Consoante as estas ideias, a dificuldade de realizar a observação minuciosa e rastrear pistas não-verbais, silêncios, pausas e a entonação da voz, itens importantes no processo terapêutico, podem prejudicar o bom andamento da terapia e da evolução do cliente, tal como, a fidedignidade da AP, que pode ser enviesada devido a percepção e aos fatores ambientais (Marasca et al., 2020; CFP, 2020b; Santana et al., 2020). Desse modo, é necessário que o profissional da psicologia esteja 
alerta e redobre sua atenção para os sinais que o cliente emitir durante o atendimento, pois alguns sujeitos podem fazer qualquer coisa na frente da câmera e esconder as demandas, enquanto outros, inibidos, podem sentir-se confortáveis na relação mediada por uma tela e apresentá-las. Essas questões são relevantes tanto durante a psicoterapia, quanto na entrevista psicológica e na testagem, para o processo de avaliação.

No mesmo sentido, no que se refere aos dados obtidos na AP online em período pandêmico, o CFP (2020b) alerta o profissional para que este se proponha a questionar se os impactos psicossociais e emocionais ocasionados pela pandemia, podem influenciar no processo avaliativo e comprometer os resultados encontrados, bem como as limitações vivenciadas pelo processo, fazendo-se necessário um olhar crítico sobre os viés e princípios éticos da benevolência e maleficência.

Do mesmo modo, é importante que ambas as partes (cliente - profissional) se inteiram da responsabilidade ética referente ao sigilo, um dos grandes desafios que a psicologia encontra. O profissional deve assegurar e lembrar ao cliente que o atendimento é sigiloso e não é permitido gravações nem fotografias durante o atendimento. Deve utilizar plataformas com criptografia de ponta, que dificultam o vazamento de dados, assim como, realizar um contrato por escrito junto ao cliente para respaldar-se.

Além disso, suscita-se a necessidade da oferta de disciplinas teórico-práticas específicas relativas ao atendimento e a AP online na grade curricular do curso de graduação em psicologia, permitindo ao acadêmico, futuro profissional, a oportunidade de realizar atendimentos na nova modalidade e debruçar na teoria e resoluções vigentes, tal como a supervisão clínica, item tão importante no tripé da psicologia - teoria-terapia-supervisão. Isto possibilita a formação de um profissional mais qualificado, que conheça as formas de atendimento, produza intervenções eficientes e obtenha resultados mais satisfatórios com seus clientes.

Soma-se a essa ideia, a carência de validação e aprovação pelo SATEPSI, de instrumentos psicométricos para utilizar no formato online, uma vez que, uma das grandes dificuldades encontradas pelos profissionais de psicologia para realização de AP online, segundo CFP (2020b), é a pequena quantidade de instrumentos válidos e favoráveis disponíveis, limitando a opção do profissional na sua atuação.

De acordo com Marasca et al. (2020), até maio de 2020 na lista de testes favoráveis para aplicação online no SATEPSI, contava com apenas 4 instrumentos e 20 instrumentos listados que permitem a aplicação informatizada, ou seja, por intermédio do computador, mas presencialmente com o avaliando. Em julho de 2021, na lista do SATEPSI possuía 12 instrumentos favoráveis para aplicação online/remota e 24 de forma informatizada

Salienta-se ainda, as falhas encontradas nas resoluções do CFP (2018; 2020a), por não deixarem claro certas questões, há ambiguidade e confusão em relação a outras no que diz respeito aos cuidados descritos pelo Código de Ética Profissional do Psicólogo (CFP, 2005). Assim, ressalta-se a necessidade de instrumentalização profissional e de estudos científicos mais abrangentes que auxiliem a suplantar as atuais limitações e a garantir resultados mais favoráveis, tal como, ensaios clínicos robustos que demonstrem a eficácia dessa nova forma de atendimento.

\section{Considerações Finais}

Em síntese, o atendimento psicológico online e a AP online vêm sendo utilizados há alguns anos de maneira experimental. Entretanto, com o avanço do uso da tecnologia e as demandas de tempo que as pessoas possuem, o atendimento remoto já é regularizado como uma ferramenta de tratamento psicológico, tendo uma procura muito grande atualmente no contexto da pandemia da Covid-19.

Diante disso e sabendo da importância desse formato para a ciência psicológica, reverbera-se a necessidade de pesquisas mais robustas nesse âmbito, incluindo pesquisas empíricas, relatos de experiências, revisão sistemática da literatura e 
ensaios clínicos randomizados, de maneira a demonstrar a eficácia e eficiência dessa forma de atendimento, além da criação de especializações lato sensu e stricto sensu sobre essa nova prática de cuidado, tal como a cobrança, pelo CFP, da supervisão clínica para os psicólogos.

\section{Referências}

American Educational Research Association, American Psychological Association, \& National Council on Measurement in Education. (2014). The Standards for educational and psychological testing. Washington: American Educational Research Association.

Bauman, Z. (2007) Tempos líquidos. $1^{\text {a }}$ Ed. Rio de Janeiro: Zahar.

Bittencourt, H. B., Rodrigues, C. C., Santos, G. L., Quadros, L. G., Mallmann, L. S., Bratkowski, P. S. \& Fedrizzi, R. I. (2020). Psicoterapia on-line: uma revisão de literatura. Diaphora: Porto Alegre v. 9 (1) jan/jun, 41-46.

Borsa, J. C. (2016). Considerações sobre a formação e prática em avaliação psicológica no Brasil. Revista Temas em Psicologia, 24(1), 131-143.

Brasil (2012). Ministério da Saúde. Secretaria de Ciência, Tecnologia e Insumos Estratégicos. Departamento de Ciência e Tecnologia. Diretrizes metodológicas: elaboração de revisão sistemática e metanálise de ensaios clínicos randomizados/ Ministério da Saúde, Secretaria de Ciência, Tecnologia e Insumos Estratégicos, Departamento de Ciência e Tecnologia. - Brasília: Editora do Ministério da Saúde.

Cohen, R. J., Swerdlink. M. E. \& Sturman, E. D (2014). Testagem e avaliação psicológicas: introdução a testes e medidas (8.ed.). Porto Alegre: AMGJ.

Conselho Federal de Psicologia (1992). Atribuições profissionais do psicólogo no Brasil. Brasília. https://site.cfp.org.br/wpcontent/uploads/2008/08/atr_prof_psicologo.pdf

Conselho Federal de Psicologia (2005). Código de Ética Profissional do psicólogo. Brasília. https://site.cfp.org.br/wp-content/uploads/2012/07/codigo-deetica-psicologia.pdf

Conselho Federal de Psicologia (2007). Cartilha de Avaliação Psicológica. Brasília. https://site.cfp.org.br/wp-content/uploads/2013/05/CartilhaAvalia\%C3\%A7\%C3\%A3o-Psicol\%C3\%B3gica.pdf

Conselho Federal de Psicologia (2012). Resolução n 0 011/2012 - Regulamenta o atendimento psicoterapêutico e outros serviços psicológicos mediados por computador e revoga a Resolução CFP n ${ }^{\circ} 012 / 2005$.

Conselho Federal de Psicologia. (2018). Resolução nº 09, de 25 de abril de 2018. Estabelece diretrizes para a realização de Avaliação Psicológica no exercício profissional da psicóloga e do psicólogo, regulamenta o Sistema de Avaliação de Testes Psicológicos - SATEPSI e revoga as Resoluções ${ }^{\circ}$ 002/2003, ${ }^{\circ}$ 006/2004 e n ${ }^{\circ}$ 005/2012 e Notas Técnicas n 01/2017 e 02/2017. Brasília. http://satepsi.cfp.org.br/docs/resolu\%c3\%a7\%c3\%a3o-cfp-n\%c2\%ba-09-2018-com anexo.pdf

Conselho Federal de Psicologia. (2019). Resolução nº, 29 de março de 2019. Institui regras para a elaboração de documentos escritos produzidos pela(o) psicóloga(o) no exercício profissional e revoga a Resolução CFP n ${ }^{\circ}$ 15/1996, a Resolução CFP nº 07/2003 e a Resolução CFP n 04/2019. https://atosoficiais.com.br/cfp/resolucao-do-exercicio-profissional-n-6-2019-institui-regras-para-a-elaboracao-de-documentos-escritos-produzidos-pela-opsicologa-o-no-exercicio-profissional-e-revoga-a-resolucao-cfp-no-15-1996-a-resolucao-cfp-no-07-2003-e-a-resolucao-cfp-no-042019? origin=instituicao $\& \mathrm{q}=$ avalia $\% \mathrm{C} 3 \% \mathrm{~A} 7 \% \mathrm{C} 3 \% \mathrm{~A} 3 \mathrm{o} \% 20 \mathrm{psicologica}$

Conselho Federal de Psicologia. (2018). Resolução $n^{\circ}$ 11, de 11 de maio de 2018. Regulamenta o atendimento psicoterapêutico e outros serviços psicológicos mediados por computador e revoga a Resolução CFP nº 011/2012. 2018.

Conselho Federal de Psicologia (2020a). Resolução nº 04, de 26 de março de 2020. Dispõe sobre regulamentação de serviços psicológicos prestados por meio de Tecnologia da Informação e da Comunicação durante a pandemia do COVID-19. Brasília: Autor. http://www.in.gov.br/en/web/dou/-/resolucao-n-4-de-26de-marcode-2020-250189333 Conselho Federal de Psicologia. (2005). Código de Ética Profissional do Psicólogo. Brasília: Autor. Recuperado de https://site.cfp.org.br/wp-content/uploads/2012/07/codigo-de-etica-psicologia.pdf

Conselho Federal de Psicologia da $3^{\text {a }}$ Região (2020). E-PSI: Conselho Federal de Psicologia disponibiliza sistema de atualização cadastral para psicólogas com endereço de e-mail desatualizado ou não cadastrado [Internet]. Salvador, BA. <http://www.crp03.org.br/e-psi-conselho-federal-de-psicologia-disponibilizasistema-de-atualizacao-cadastral-para-psicologascom-endereco-de-e-email-desatualizado-ou-nao-cadastrado/>

Conselho Federal de Psicologia (2020b). Cartilha de boas práticas para avaliação psicológica em contextos de pandemia. Brasília: CFP.

Evangelista, P. E. R. \& Cardoso, C. L (2020) Aconselhamento psicológico fenomenológico-existencial online como possibilidade de atenção psicológica durante a pandemia de Covid-19. Perspectivas em Psicologia, Uberlândia. 24(2), 159-3.

Fieldman, R. S (2015). Introdução a Psicologia Clínica (10.ed.). Porto Alegre: AMGH.

Gomes, S. G. S. (2011). Evolução histórica da ead. E-Tec Brasil-Tópicos em Educação a Distância. http://ftp. comprasnet. se. gov. br/sead/licitacoes/Pregoes2011/PE091/Anexos/Evento s_modulo_I/topico_ead/Aula_02.pdf.

Gouveia, V. V. (2018). Formação em avaliação psicológica: situação, desafios e diretrizes. Psicologia: Ciência e Profissão, 38 , 74-86.

Hutz, C. S., Bandeira, A. D. R. \& Trentini, V. M. (organizadores) (2015). Psicometria. Porto Alegre: Artmed. 
Luxton, D. D., Pruitt, L. D., \& Osenbach, J. E. (2014). Best practices for remote psychological assessment via telehealth technologies. Professional Psychology: Research and Practice, 45(1), 27. http://dx.doi.org/10.1037/a0034547

Lustgarten, S. D., \& Elhai, J. D. (2018). Technology use in mental health practice and research: legal and ethical risks. Clinical Psychology: Science and Practice, 25(2), 1-10. http://dx.doi.org/10.1111/cpsp.12234

Marasca, A. R., Yates, D. B., Schneider, A. M. A., Feijó, L. P. \& Bandeira, D. R. (2020). Avaliação psicológica online: considerações a partir da pandemia do novo coronavírus (COVID-19) para a prática e o ensino no contexto a distância. Estudos de Psicologia (Campinas), 37, e200085, 1-11. http://doi.org/10.1590/1982-0275202037e200085.

McCord, C., Bernhard, P., Walsh, M., Rosner, C., \& Console, K. (2020). A consolidated model for telepsychology practice. Journal of Clinical Psychology, 76(6), 1060-1082. https://dx.doi.org/10.1002/jclp.22954

Ministério da Saúde (2020). Recomendações de proteção aos trabalhadores dos serviços de saúde no atendimento de COVID-19 e outras síndromes gripais Recuperado https://www.saude.go.gov.br/files/banner_coronavirus/GuiaMS-Recomendacoesdeprotecaotrabalhadores-COVID-19.pdf

Oliveira, E. N., Costa, M. S. A., Nascimento, P. I. da F. V. do, Rodrigues, C. S., Andrade, C. S. G. de, Mendonça, J. M. F., Pinto, M. R., França, S. da S. \& Lima, G. F. (2020). Com a palavra os profissionais de saúde na linha de frente do combate à COVID-19. Research, Society and Development, 9(8), e30985145. https://doi.org/10.33448/rsd-v9i8.5145

Ornell, F., Schuch, J. B., Sordi, A. O., \& Kessler, F. H. P. (2020). Medo pandêmico" e COVID-19: ônus e estratégias de saúde mental. Revista Brasileira de Psiquiatria, 42(3), 232-235.

Pasquali, L (2016). Os testes psicológicos no Brasil. In L. Pasquali (Ed.) TEP-Técnicas de Exame Psicológico: Os fundamentos (2a ed.) (pp. 201- 220). São Paulo: Vetor Editora.

Pereira, M. M., Trajano, A. R. C., Caversan, H. A. C.; Moreira, S. P. \& Tibiriça, V. A. (2021) Plantão psicológico: a prática do acolhimento online durante a pandemia da COVID- 19. Revista De Extensão Da UPE, 6 (Especial COVID-19), 39-51. https://www.revistaextensao.upe.br/index.php/reupe/article/view/96

Salek, L., \& Moser, A. (2020). Atendimento psicológico online. Revista Científica Sophia, 1(1), 99-110. http://ojs.avantis.edu.br/index.php/sophia/article/view/111

Sampaio, R. F., \& Mancini, M. C. (2007). Estudos de revisão sistemática: um guia para síntese criteriosa da evidência científica. Brazilian Journal of Physical Therapy, 11, 83-89.

Santana, S. R., Girard, C. D. T., Costa, L. C. A., Girard, C. M. T. \& Costa, D. J. E. (2020). Os desafios dos serviços psicológicos mediados pelas TIC no contexto da pandemia do coronavírus 2019-2020. Periódicos da UFCA. 6(1), 59-71.

Siegmund, G., \& Lisboa, C. (2015). Orientação psicológica online: percepção dos profissionais sobre a relação com os clientes. Psicologia: Ciência e Profissão, 35(1), 168-181.

Schmidt, B., Crepaldi, M. A., Bolze, S. D. A., Neiva-Silva, L., \& Demenech, L. M. (2020). Saúde mental e intervenções psicológicas diante da pandemia do novo coronavírus (COVID-19). Estudos de Psicologia (Campinas), 37, e200063. https://dx.doi.org/10.1590/1982-0275202037e200063

Schmidt, B., Silva, I. M., Pieta, M. A. M., Crepaldi, M. A., Wagner, A. (2020). Terapia online com casais e famílias: Prática e formação na pandemia de Covid-19. Psicologia: Ciência e Profissão, 40, 1-15. https://doi.org/10.1590/1982-3703003243001

Schmidt, B., Palazzi, A. \& Piccinini, C. A. (2020). Entrevista Online: Potencialidades e desafios para coletas de dados no contexto da pandemia de COVID 19. REFACS.

Silva, M. de O., \& Ribeiro, A. da S. (2020). Enfermeiros na linha de frente do combate à COVID-19: saúde profissional e assistência ao usuário. Research, Society and Development, 9(8), e172985241. https://doi.org/10.33448/rsd-v9i8.5241

Souza, M. R. de; Ribeiro, A. L. P. (2009) Revisão sistemática e Meta-análise de estudos de diagnóstico e prognóstico: um tutorial. Arq. Bra. Cariol; 92 (3), 241-251.

Ulkovski, E. P., Silva, L. P. D., \& Ribeiro, A. B. (2017). Atendimento psicológico online: perspectivas e desafios atuais da psicoterapia. Revista de Iniciação Científica da Universidade Vale do Rio Verde, 7(1), 59-68.

Viana, D. M. (2020). Atendimento psicológico online no contexto da pandemia de covid-19. Cadernos Esp Ceará, jan-jun.; 14(1), 74-79 ISSN: 18087329/1809-0893. https://cadernos.esp.ce.gov.br/index.php/cadernos/article/view/399

Wang, C., Pan, R., Wan, X., Tan, Y., Xu, L., Ho, C. S., \& Ho, R. C. (2020). Immediate psychological responses and associated factors during the initial stage of the 2019 coronavirus disease (COVID-19) epidemic among the general population in china. International Journal of Environmental Research and Public Health, 175), 1729. https://www.ncbi.nlm.nih.gov/pmc/articles/PMC7084952/

Xiao, C. (2020). A novel approach of consultation on 2019 novel coronavirus (COVID-19)-related psychological and mental problems: structured letter therapy. Psychiatry Investigation, 17(2), 175-176. https://www.ncbi.nlm.nih.gov/pmc/articles/PMC7047000/Colocar espaço entre uma referência e outra Lembre-se que usamos a norma APA. (fonte TNR 8 - espaço simples -justificado) 